\title{
FORMACIÓN DE INVESTIGADORES EN EL CONTEXTO DE PROYECTOS COLABORATIVOS: EXPERIENCIAS EN EL INSTITUTO DE MEDICINA TROPICAL “ALEXANDER VON HUMBOLDT”, UNIVERSIDAD PERUANA CAYETANO HEREDIA
}

\author{
Eduardo Gotuzzo ${ }^{1,2, a}$, Elsa González ${ }^{1,2, b}$, Kristien Verdonck $k^{1,3, c}$
}

\begin{abstract}
RESUMEN
La investigación constituye un elemento fundamental de desarrollo humano y social. Bajo esta perspectiva, supone desafíos y oportunidades particulares para los llamados países "en vías de desarrollo". Una aproximación a tales desafíos y oportunidades se desprende del análisis de dos actividades interrelacionadas: la formación de nuevos investigadores y el desarrollo de investigación con instituciones o investigadores externos a la propia institución ("investigación colaborativa"). Ambas actividades son esenciales en la consolidación, ampliación y actualización de las capacidades institucionales de producción científica. Presentamos aquí las experiencias del Instituto de Medicina Tropical "Alexander von Humboldt" de la Universidad Peruana Cayetano Heredia con relación a la formación de investigadores; discutimos los cuatro elementos que consideramos clave en dicho proceso: promoción de entornos estimulantes para investigación, identificación proactiva de becarios, asesorías complementarias y consolidación de redes; y analizamos tres modelos de colaboración internacional exitosos en la formación de nuevos investigadores bajo distintas aproximaciones institucionales.
\end{abstract}

Palabras clave: Apoyo a la formación profesional; Cooperación internacional; Mentores; Investigación biomédica; Perú (fuente: DeCS BIREME).

\section{RESEARCHERS TRAINING IN THE CONTEXT OF THE COLLABORATIVE PROJECTS: EXPERIENCES OF INSTITUTO DE MEDICINA TROPICAL “ALEXANDER VON HUMBOLT”, UNIVERSIDAD PERUANA CAYETANO HEREDIA}

\begin{abstract}
Research is a main element for human and social development. Under this point of view, it involves particular challenges and opportunities for the so-called "developing countries". An approach for those challenges and opportunities comes from the analysis of two interrelated activities; the training of new researchers and the research development with institutions or researchers which are external to the institution ("collaborative research"). Both activities are essential for the consolidation, widening and updating of the institutional capabilities for scientific production. We present here the experiences of the Instituto de Medicina Tropical "Alexander von Humboldt" of the Universidad Peruana Cayetano Heredia, in relation to the training of new researchers, we discuss the four elements we consider key for this process; the promotion of stimulating environments for research, the proactive identification of fellows, the complementary advice and networks consolidation; and we analyze three successful models of international collaboration for the training of new researchers under different institutional approaches.
\end{abstract}

Key words: Training support; International cooperation; Mentors; Biomedical research; Peru (source: MeSH NLM).

\section{INTRODUCCIÓN}

La investigación es función inherente de las universidades. En el Perú, adicionalmente y de acuerdo con la ley, resulta obligatoria ${ }^{(1)}$. Por su contribución al proceso de formación de investigadores, los modelos de investigación colaborativa son determinantes para el fortalecimiento de las universidades. En este artículo, desde la perspectiva de un instituto universitario, se resumen, los que en nuestra opinión, constituyen los aspectos claves de investigación colaborativa para la formación de investigadores.

\footnotetext{
Instituto de Medicina Tropical Alexander von Humboldt, Universidad Peruana Cayetano Heredia. Lima, Perú.

2 Facultad de Medicina, Universidad Peruana Cayetano Heredia. Lima, Perú.

3 Instituto de Medicina Tropical. Amberes, Bélgica.

a Médico, especialista en enfermedades infecciosas y tropicales; ${ }^{b}$ Médico; ${ }^{c}$ Médico, doctor en ciencias médicas.
} 


\section{INVESTIGACIÓN COLABORATIVA COMO MODELO PREDOMINANTE PARA LA FORMACIÓN DE INVESTIGADORES}

La colaboración internacional es un modelo de trabajo científico predominante que se define en torno a objetivos comunes de investigación. La confianza y la expectativa sobre los beneficios potenciales para todas las partes involucradas son los elementos centrales que subyacen a las investigaciones colaborativas y son incompatibles con perspectivas unilaterales ${ }^{(2)}$. Por lo tanto, situaciones caracterizadas por la falta de expectativas respecto a la potencial contribución del otro colaborador, o en las que se emplea a una de las partes como sujeto de un estudio cuyos resultados serán de beneficio exclusivo de la otra ${ }^{(3,4)}$, no representan investigaciones colaborativas sino de participación simultánea.

Las crecientes facilidades para las comunicaciones han aumentado la participación e interés de la comunidad científica en general hacia las investigaciones colaborativas internacionales ${ }^{(5)}$. La colaboración entre diversos grupos de investigadores es reconocida como una característica esencial de la investigación del siglo XXI ${ }^{(6)}$. Aunque se espera coincidencias entre las partes involucradas en cuanto al planteamiento de los objetivos científicos y los principios comunes de trabajo, las particularidades de sus respectivos contextos y experiencias condicionan diferencias tanto en los estilos como en las prioridades del trabajo ${ }^{(2,7-9)}$. El proceso de exposición y enfrentamiento de estas diferencias no está exento de dificultades y frustraciones, pero suele tener, para ambas partes, una repercusión positiva que se proyecta a múltiples esferas ${ }^{(10-12)}$.

En contextos con poca disponibilidad de financiamiento interno para investigación, la mayoría de estas actividades y los programas que facilitan opciones de entrenamiento en investigación, se ejecutan en el marco de investigaciones colaborativas. Este hecho repercute en la producción científica de las instituciones; como ejemplo, el $75 \%$ de las publicaciones recientes del Instituto de Medicina Tropical Alexander von Humboldt (IMTAvH) incluyen a coautores extranjeros (Figura 1).

\section{ELEMENTOS CLAVE EN LA FORMACIÓN DE INVESTIGADORES}

La Tabla 1 sintetiza los que en nuestra opinión, constituyen los elementos clave de los procesos de formación de investigadores, bajo una perspectiva de fortalecimiento institucional. Aunque estos elementos se prestan a un planteamiento de situación ideal, indudablemente existe un proceso de retroalimentación bilateral entre cada uno de ellos y el de formación de investigadores.

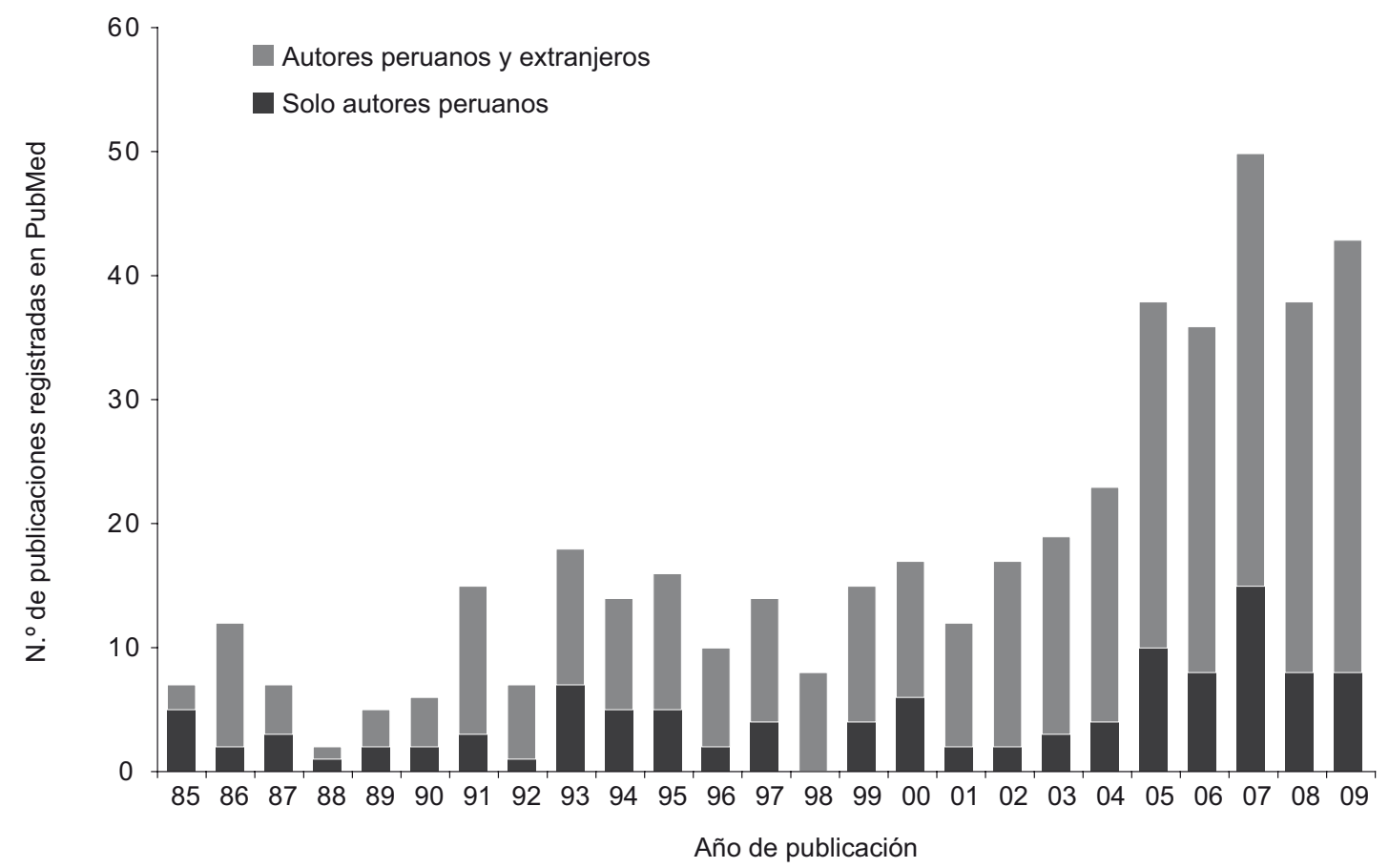

Figura 1. Instituto de Medicina Tropical Alexander von Humboldt: tendencias en las publicaciones científicas colaborativas (1985-2009). 
Tabla 1. Elementos clave en los procesos de formación de investigadores.

\begin{tabular}{l}
\hline \multicolumn{1}{c}{ Elementos clave } \\
\hline 1. Promoción de entornos estimulantes \\
\hline 2. Identificación proactiva de becarios \\
3. Mentorías complementarias \\
4. Consolidación de redes \\
\hline
\end{tabular}

\section{PROMOCIÓN DE ENTORNOS ESTIMULANTES PARA INVESTIGACIÓN}

Un entorno estimulante, constituido por las personas que conforman la institución, su acervo y sus principios, tiene efectos positivos en las vocaciones y en las carreras de investigación. Estos entornos pueden darse bajo muy diversas condiciones, pero finalmente, propician miradas abiertas, críticas y, al mismo tiempo, con sentido de responsabilidad hacia el mundo en sus diversidades ${ }^{(13-17)}$.

La Universidad Peruana Cayetano Heredia (UPCH), es la institución local con mayor producción científica en salud en Perú ${ }^{(18)}$. La investigación biomédica ha sido propiciada activamente desde su fundación bajo una perspectiva atenta a los aspectos científicos y también a aquellos de responsabilidad social para el desarrollo, que les corresponde promover y asumir a las universidades ${ }^{(19)}$. El reconocido liderazgo de figuras que en la mayoría de casos corresponden a científicos y humanistas, como los doctores Hurtado, Monge, Cazorla, Lumbreras, Torres, León Barúa, Pretell, Guerra-García, fue construyendo un ambiente proactivo y motivador para la investigación y la formación de investigadores.

Ambientes como estos resultan propicios para que investigadores, con distintos grados de experiencia, interactúen con estudiantes de pregrado y posgrado; la participación conjunta de todos ellos vitaliza, fortalece y consolida estos ambientes ${ }^{(20-27)}$. En nuestra opinión, estos entornos son fundamentales para que los jóvenes elementos puedan trabajar creativamente, familiarizarse con una visión de investigación que concilie intereses científicos y extracientíficos, e irse perfilando como investigadores.

Con cierta frecuencia podemos reconocer a estudiantes muy talentosos que inician desde el pregrado una promisoria trayectoria de investigación ${ }^{(28)}$. Ya graduados, la mayoría de estos estudiantes destacados continúa publicando de manera activa sus investigaciones y opta por participar de maestrías o doctorados.
Evidentemente, los entornos se nutren también de acciones y recursos específicos ${ }^{(29,30)}$. Por ejemplo, con el objetivo que todos los alumnos de la UPCH se familiaricen con los procesos de investigación, la tesis se mantiene como una exigencia para la graduación de pregrado y posgrado ${ }^{(31)}$. Adicionalmente, la UPCH fue la primera universidad peruana en crear un Vicerrectorado de Investigación, decisión que permitió poner en práctica políticas institucionales de promoción, entre ellas: (i) la generación de fondos concursables para estimular el desarrollo de proyectos de investigación que involucren simultáneamente a docentes y alumnos y, (ii) la generación de las becas de retorno, un programa orientado a la repatriación de científicos talentosos formados internacionalmente, con el objetivo de impulsar un área determinada del conocimiento ${ }^{(32)}$. Como efecto de esas políticas institucionales, en los últimos cinco años los proyectos de investigación colaborativa, a la par que las opciones de maestrías y doctorados en el Perú y el extranjero para jóvenes profesionales de la $\mathrm{UPCH}$, se han incrementado sustancialmente.

\section{IDENTIFICACIÓN PROACTIVA DE BECARIOS}

Independientemente del entorno específico, una carrera exitosa en investigación presupone altas cualidades personales y profesionales, dentro de un marco muy variado de perfiles individuales ${ }^{(11,12,33)}$. El entrenamiento de un investigador demanda el continuo equilibrio entre la enseñanza y el aprendizaje, el orgullo y la humildad, la firmeza y la adaptabilidad (11).

Un profesional decidido a hacer investigación en un país como el Perú debe poder equilibrar constructivamente el afán por la excelencia científica con la disponibilidad reducida de recursos; los procesos de integración en una institución local y el contacto con el contexto nacional $(11,34,35)$. Al mismo tiempo debe estar en capacidad de entender y demostrar que, precisamente en países como el Perú y en paralelo al hecho tan reconocido de las limitaciones locales, existen también enormes oportunidades ${ }^{(36)}$ y necesidades que pueden ser identificadas y construidas, al menos parcialmente, a partir de tales limitaciones. En muchos casos esta coyuntura puede ser canalizada de forma enriquecedora y ventajosa para la labor de investigación y para la formación de un investigador consolidado.

Para que un proceso de selección cumpla con identificar a los mejores candidatos, el grupo de potenciales interesados debe ser suficientemente amplio ${ }^{(6)}$. Como se sugería en la sección anterior, la amplitud del grupo de potenciales interesados se relaciona con aspectos del entorno. La definición de los "mejores candidatos" no depende exclusivamente de las competencias 
académicas o científicas individuales, pues debe considerar otros factores, como la voluntad personal de retorno al país o a la institución de origen al término del entrenamiento ${ }^{(10)}$. Sin desconocer la contribución que un profesional puede realizar al país al decidir continuar su carrera desde el exterior ${ }^{(37)}$, está claro que esa situación no corresponde a las expectativas institucionales que determinan la selección de un becario para un entrenamiento específico. En nuestra institución, se han añadido a los criterios de desempeño académico, las capacidades de liderazgo y la disposición percibida a mediano plazo para trabajar por el desarrollo de la institución ("nivel de identificación") en la selección de becas de doctorado.

La transparencia respecto a las expectativas y compromisos mutuos entre la institución y el becario es un aspecto que debe ser considerado tempranamente, e implementado a lo largo de todo el proceso de selección. Una comunicación explícita en tal sentido puede evitar malentendidos que se agravan con el tiempo y que aumentan el riesgo de fuga de recursos entrenados.

\section{ASESORIASS COMPLEMENTARIAS}

Dado que los entrenamientos avanzados para investigadores a través de proyectos de colaboración suelen incluir estadías alternativas en la institución extranjera y en la institución local, con frecuencia se requiere un asesor por cada una de las dos instituciones. Habitualmente, ambos asesores mantienen un nivel de responsabilidad final similar, con diferencias en las respectivas áreas de competencia.

En el mejor escenario, el becario se convierte en un investigador flexible, acostumbrado a investigación multidisciplinaria, e integrado en redes internacionales. Estas son características de un investigador moderno y son apreciadas extendidamente, también en países industrializados donde continúan en aumento los cuestionamientos al modelo de hiperespecialización para el entrenamiento de investigadores, tan promovido durante la década pasada ${ }^{(6,11,33)}$. En nuestra opinión, el problema podría estar no en la hiperespecialización propiamente, sino en la reducida capacidad de diálogo e interacción con investigadores de otras disciplinas que suele acompañar a tal hiperespecialización.

La buena relación con el/los asesor/es es crucial para el éxito de la formación del investigador. Los mentores pueden tener estilos personales muy distintos y, en su complementariedad con aquellos del becario, puede residir el potencial de éxito o fracaso de tal relación. El entrenamiento debe permitir consolidar las dotes naturales y controlar las desventajas personales del becario, aprendizaje que se logra en gran medida con apoyo del mentor. Un buen mentor no solo motiva, acompaña y supervisa durante el entrenamiento, sino que se mantiene vinculado para ayudar a abrir puertas y facilitar una carrera como investigador autónomo ${ }^{(6,11,12,33)}$. Idealmente, el asesor y el becario deberían seleccionarse mutuamente y en toda libertad ${ }^{(12,33)}$. En la realidad, esto es muy difícil dado que, con frecuencia, las particularidades del programa que otorga la beca ( $p$. ej. condiciones, monto y duración de la beca, apoyo luego del entrenamiento, temas prioritarios) afectan las opciones de los potenciales asesores extranjeros. Paralelamente, el número relativamente reducido de profesores locales experimentados en investigación limita las opciones.

Es recomendable que el becario mantenga una comunicación constante con ambos asesores y, al mismo tiempo, permita un canal de comunicación entre ellos. Por otra parte, los asesores deben facilitar que, sin descuidar sus actividades de entrenamiento en investigación, el becario asuma progresivamente otras que amplíen sus posibilidades de integración a la institución local ${ }^{(34,38)}$. Las actividades de enseñanza son un gran recurso de aprendizaje; por ello, consideramos recomendable facilitar que los becarios con interés en investigación y docencia se involucren en la enseñanza, en forma focalizada pero progresiva, bajo guía de sus mentores. Bien encaminadas, estas actividades pueden fortalecer programas académicos vigentes y contribuir con el desarrollo de otros de potencial interés para el país.

\section{CONSOLIDACIÓN DE REDES}

En instituciones pequeñas o localizadas en países en vías de desarrollo, la participación en redes es esencial para prevenir el aislamiento y la fuga de cerebros ${ }^{(10,12,35-38)}$.

Aunque el incremento de alianzas internacionales tiene consecuencias muy positivas que ya han sido comentadas, genera también algunos retos. Existe una necesidad cada vez más grande de coordinación para prevenir la duplicación, la fragmentación y la competencia poco saludable entre diferentes proyectos ${ }^{36}$. Cada agencia financiadora impone sus propios reglamentos, cuyo seguimiento impone una carga administrativa creciente. Finalmente, un gran reto, tal vez no muy aparente, es el de mantener las prioridades locales de investigación en la agenda internacional ${ }^{(3,39-42)}$.

En este contexto, no solo se debería apuntar a mantener e impulsar las redes norte-sur, las más frecuentes en nuestro medio, sino también fortalecer las colaboraciones a nivel regional ${ }^{(43-46)} \mathrm{y}$, muy particularmente, con otras 
instituciones peruanas. Las redes regionales o sur-sur, han demostrado ser particularmente efectivas para un uso más eficiente de recursos, la creación de oportunidades de carrera científica y acciones de retención de masa crítica ${ }^{(46-48)}$. Otros países en América Latina, como Brasil, Chile, México y Argentina vienen promoviendo tales colaboraciones, y constituyen modelos interesantes que multiplican espacios y oportunidades para sus nuevos investigadores. Las redes internas o relaciones de colaboración entre instituciones peruanas, deberían ser vistas con similar o mayor interés, en la medida que podrían contribuir a consolidar las capacidades locales de investigación y articularlas hacia temas que en ese ámbito tienen especial relevancia. Idealmente, la comunidad de investigadores debería poder contribuir con las necesidades, evidentes o implícitas, de su propia sociedad ${ }^{34,42,49)}$. Por otra parte, debería comunicar los resultados de investigación a las instancias correspondientes del sector para que, en los casos pertinentes, fueran progresivamente considerados en las condiciones de rutina. Fortalecer estas dos actividades sitúa a los grupos de investigación en mejor posición para proporcionar la evidencia localmente necesaria ${ }^{(43,45)}$.

Lamentablemente, un gran limitante para el desarrollo de redes nacionales de investigación es que todavía existen muy pocos incentivos tangibles que las promuevan. Desde un punto de vista estrictamente financiero, invertir esfuerzos en promover colaboraciones con socios extranjeros representa una opción mucho más atrayente, lo que condiciona que diferentes grupos de investigadores peruanos suelan percibirse como posibles competidores y no como potenciales colaboradores. Consideramos que este es uno de los mayores desafíos que deberán ir asumiendo los nuevos investigadores de nuestro medio.

\section{BREVE DESCRIPCIÓN DE MODELOS DE PROYECTOS COLABORATIVOS Y FORMACIÓN DE INVESTIGADORES EN EL IMTAVH}

En el IMTAvH, los proyectos de investigación colaborativa con instituciones internacionales que permiten la formación de investigadores corresponden fundamentalmente a dos modelos (Tabla 2): a) programas de entrenamiento en investigación (research training grants) y b) programas de fortalecimiento institucional.

El programa de colaboración con el Instituto de Medicina Tropical de Amberes, Bélgica (ITG) corresponde al modelo de fortalecimiento institucional. Con apoyo de la Cooperación Belga, el objetivo específico de este programa es el fortalecimiento del IMTAvH como centro de excelencia en la investigación clínica de las enfermedades infecciosas y tropicales. La evolución general del programa, determinada por el alcance de hitos significativos en sus etapas principales, se resume en la Figura 2. En la actualidad, la formación de investigadores es el eje central del programa y permite ocho posiciones de doctorado. Este programa es uno de los más importantes para el IMTAvH, como se refleja a partir de la producción científica conjunta entre ambas instituciones: en el período 1985-2009, 450 publicaciones registradas en PubMed incluyeron, por lo menos, un autor del IMTAvH; de las 450, 68 (15\%) listaron también entre los autores a un investigador del ITG. En los últimos cinco años, las publicaciones con coautores del ITG representaron 22\% (46/205) con 38 investigadores del ITG como coautores de publicaciones del IMTAvH. A partir de la colaboración con el ITG se han generado contactos con otros institutos y universidades en Europa: las Universidades de Amberes y de Leuven en Bélgica; la Universidad de Leiden en Holanda; la Universidad de Leeds y el Imperial College de Londres en Ingla-

Tabla 2. Comparación general de programas de entrenamiento en investigación y programas de fortalecimiento institucional.

\begin{tabular}{|c|c|c|}
\hline & $\begin{array}{c}\text { Programas de Entrenamiento en } \\
\text { Investigación }\end{array}$ & $\begin{array}{c}\text { Programas de Fortalecimiento } \\
\text { Institucional }\end{array}$ \\
\hline Socio institucional principal & Universidad de Estados Unidos & Instituto de Investigación Europeo \\
\hline Áreas temáticas de interés & Una o dos, usualmente predefinidas. & $\begin{array}{l}\text { Múltiples, habitualmente definidas } \\
\text { por consenso entre las instituciones } \\
\text { participantes }\end{array}$ \\
\hline Tipos de entrenamiento financiados & $\begin{array}{l}\text { Múltiples (cursos cortos, maestrías, } \\
\text { doctorados) }\end{array}$ & Múltiples, con énfasis en doctorados \\
\hline Lugares de programas de entrenamiento & En las instituciones colaboradoras & En las instituciones colaboradoras \\
\hline $\begin{array}{l}\text { Formato de entrenamiento principal en } \\
\text { modalidades fuera del país }\end{array}$ & $\begin{array}{l}\text { Tiempo completo fuera del país } \\
\text { (al menos inicialmente) }\end{array}$ & Formato "sándwich" \\
\hline $\begin{array}{l}\text { Retención del becario en la institución } \\
\text { nacional postentrenamiento }\end{array}$ & Situación ideal, no necesaria & Situación ideal, necesaria \\
\hline
\end{tabular}


terra; el Instituto Karolinska en Suecia; la Universidad de Florencia en Italia. Así mismo, se ha fortalecido el apoyo del "Programa Especial de Investigaciones y Enseñanzas sobre Enfermedades Tropicales (TDR), patrocinado por
UNICEF, PNUD, Banco Mundial y la Organización Mundial de la Salud (OMS), Organización Panamericana de la Salud (OPS), Foundation for Innovative New Diagnostics (FIND), entre otros.
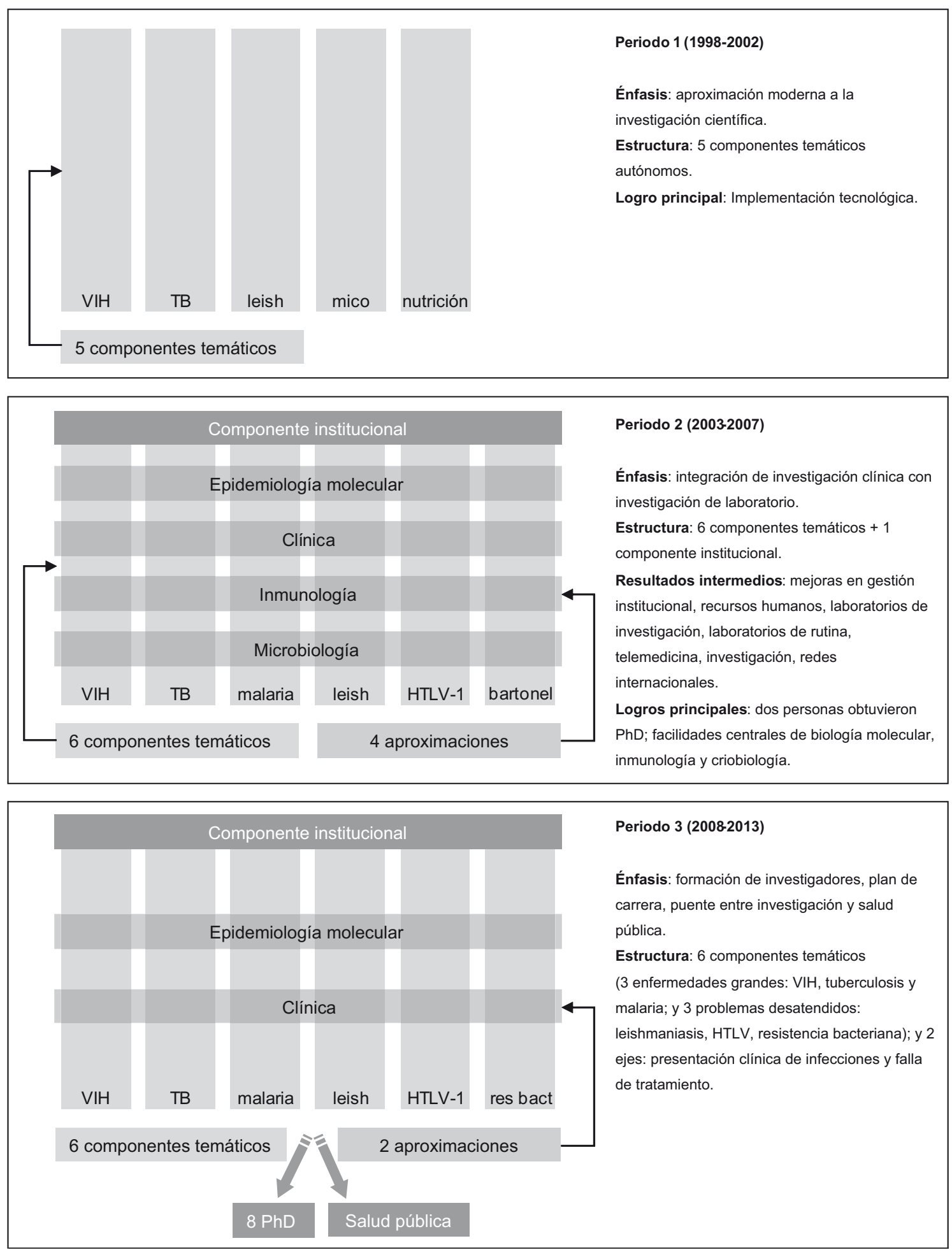

Figura 2. Evolución del Proyecto de Fortalecimiento Institucional entre el Instituto de Medicina Tropical de Amberes y el Instituto de Medicina Tropical "Alexander von Humboldt” (1998-2013). 
Por su parte, el Baylor-Cayetano Heredia Training Program in Global Infectious Diseases, realizado en colaboración con el Dr. Clinton White, actualmente en la Universidad de Texas-Galveston, con financiamiento del Fogarty International Center, es un modelo interesante que enfatiza el desarrollo de carreras profesionales progresivamente autónomas entre jóvenes investigadores de elevadas calificaciones técnicas. A través de este proyecto hemos podido repatriar a tres jóvenes profesionales entrenados en Estados Unidos; uno de ellos se desempeña actualmente como jefe del laboratorio de inmunología del IMTAvH; la segunda persona es una dinámica investigadora que lidera un proyecto de enfermedades entéricas y acaba de ganar un proyecto de la Fundación Gates; la tercera persona está completando su doctorado y en paralelo es la coordinadora actual del curso internacional Hugo Lumbreras para alumnos de medicina del exterior. Estos tres profesionales han desarrollado más de 25 publicaciones indexadas en los últimos cinco años.

Otro modelo interesante por su énfasis en la amplificación del aprendizaje individual hacia entornos más amplios y por articular esfuerzos de investigación entre los sectores público y académico es el proyecto ICOHRTA Perú (Peru ICOHRTA Network for AIDS/TB Research Training), iniciativa de la UPCH en colaboración con la Universidad de Washington en Seattle y la Universidad de Alabama en Birmingham. En colaboración con las universidades norteamericanas ya mencionadas, el Naval Medical Research Center Detachment en Lima, Perú, y otros programas de entrenamiento y unidades académicas de la UPCH, el proyecto ICOHRTA Perú desarrolló recientemente el Diplomado de Investigación Operativa con mención en (i) tuberculosis y VIH y, (ii) salud global. Se decidió enfatizar el área de investigación operativa por ser de uso todavía limitado en el Perú y por su gran potencial para la evaluación e implementación de intervenciones relevantes para el control de enfermedades como tuberculosis y VIH/SIDA. En la primera edición del programa participaron como estudiantes 24 profesionales del sector público de salud; como docentes, los becarios del programa que en años anteriores habían recibido entrenamiento internacional en investigación operativa y varios jóvenes becarios de otros programas de entrenamiento en investigación tuvieron un rol muy importante. En la actualidad, se mantiene un vínculo de trabajo entre integrantes de ambos grupos a través de proyectos específicos de investigación operativa, en una interacción valiosa para ambas partes.

En resumen, bajo enfoques diferentes los programas indicados representan modelos interesantes de cooperación internacional de impacto positivo. Este impacto ha sido aquí descrito fundamentalmente a
Tabla 3. Instituto de Medicina Tropical "Alexander von Humboldt": Evolución del personal contratado para proyectos de investigación (2004-2010).

\begin{tabular}{|c|c|c|c|c|}
\hline \multirow{2}{*}{ Personal } & \multirow{2}{*}{2004} & \multirow{2}{*}{2008} & \multicolumn{2}{|c|}{2010} \\
\hline & & & Temporal & Fijo \\
\hline Médicos & 13 & 27 & 1 & 31 \\
\hline Biólogos & 12 & 19 & 1 & 25 \\
\hline Enfermeras & 8 & 9 & 3 & 15 \\
\hline Tecnólogos de laboratorio & 2 & 7 & 1 & 10 \\
\hline Técnicos de enfermería & 9 & 10 & 1 & 14 \\
\hline Técnicos de laboratorio & 3 & 4 & 0 & 10 \\
\hline Administradores & 1 & 2 & 0 & 2 \\
\hline $\begin{array}{l}\text { Apoyo administrativo / } \\
\text { secretarial }\end{array}$ & 3 & 3 & 0 & 8 \\
\hline Otros & 0 & 4 & 1 & 20 \\
\hline Total & 51 & 85 & 143 & \\
\hline
\end{tabular}

través de la capacitación de profesionales jóvenes como nuevos investigadores, pero se extiende a la generación de empleo (Tabla 3), la mejora de la infraestructura y la elevación de los estándares de calidad. Estos aportes repercuten positivamente en actividades de diagnóstico y atención a los pacientes con enfermedades infecciosas y tropicales y en las actividades institucionales de docencia y capacitación (en nuestro caso, los programas de especialización en medicina, la Maestría de Control de Enfermedades Infecciosas y Tropicales, los cursos Gorgas, el Diplomado de Investigación Operativa y el curso internacional Hugo Lumbreras).

\section{COMENTARIOS FINALES}

Para ilustrar que a lo largo del tiempo las condiciones relacionadas con la formación de investigadores pueden variar ${ }^{(50)}$, pero que esencialmente las prioridades y principios se mantienen, nos remontaremos a mediados de los años cincuenta. Por entonces, un talentoso grupo de jóvenes profesionales y estudiantes san marquinos, vivamente interesados en el estudio de las enfermedades transmisibles y tropicales, fueron canalizando y fortaleciendo tal interés bajo guía cercana de los doctores Enrique Encinas Franco y Hugo Pesce. Poco tiempo después, y por intervención directa del Dr. Encinas, algunos integrantes del referido grupo, entre ellos el Dr. Lumbreras, viajaron para continuar su entrenamiento en el Instituto de Medicina Tropical de Hamburgo bajo mentoría del Profesor Ernst G. Nauck. Como resultado de gestiones que involucraron fundamentalmente al Dr. Lumbreras durante su estadía en Hamburgo, el Dr. Nauck visitó Lima en 1957, en el marco de una misión del gobierno alemán orientada 
a evaluar la pertinencia de financiar un Instituto de Medicina Tropical en Lima ${ }^{(51)}$. Los resultados de dicha visita fueron favorables para la iniciativa original $y$ abrieron puertas para opciones subsecuentes de entrenamiento a otros miembros de tal grupo. Si bien circunstancias posteriores relacionadas con la renuncia masiva de 450 profesores de la Facultad de Medicina de la Universidad Mayor de San Marcos en julio de 1961, impusieron nuevos desafíos y oportunidades, la experiencia en su conjunto fue indudablemente valiosa para el país, pues contribuyó a la creación del Instituto de Medicina Tropical "Daniel A. Carrión" de la Universidad Nacional Mayor de San Marcos (1963) y del IMTAvH (1968).

\section{AGRADECIMIENTOS}

A la Cooperación Belga a través del proyecto de colaboración institucional entre el Instituto de Medicina Tropical de Amberes, Bélgica y el Instituto de Medicina Tropical Alexander von Humboldt de la Universidad Peruana Cayetano Heredia (Programa FA-3) y al programa ICOHRTA Perú (Peru ICOHRTA Network for AIDS/TB Research Training NIH Research Grant \# U2R TW007368) de la Universidad Peruana Cayetano Heredia, financiado por el Fogarty International Center, National Institutes of Health.

\section{Conflictos de Interés}

Los autores declaran no tener conflictos de interés en la publicación de este artículo.

\section{REFERENCIAS BIBLIOGRÁFICAS}

1. Perú, Congreso de la República. Ley N ${ }^{\circ}$ 23733: Ley universitaria. Lima: Congreso de la República; 1983.

2. Schulz-Baldes A, Vayena E, Biller-Andorno N. Sharing benefits in international health research. Research-capacity building as an example of an indirect collective benefit. EMBO Rep. 2007;8(1):8-13.

3. Lee K, Mills A. Strengthening governance for global health research. BMJ. 2000;321(7264):775-76.

4. Snyder J. Multiple forms of exploitation in international research: the need for multiple standards of fairness. Am J Bioeth. 2010;10(6):40-41.

5. Glew RH. Promoting collaborations between biomedical scholars in the U.S. and sub-Saharan Africa. Exp Biol Med (Maywood). 2008;233(3):277-85.

6. Lenfant $\mathbf{C}$. Training the next generation of biomedical researchers: challenges and opportunities. Circulation. 2000;102(4):368-70.

7. Wall SG. Epidemiology in developing countries--some experiences from collaboration across disciplines and cultures. Scand J Soc Med Suppl. 1991;46:25-32.
8. Matee MI, Manyando C, Ndumbe PM, Corrah T, Jaoko WG, Kitua AY, et al. European and developing countries clinical trials partnership (EDCTP): the path towards a true partnership. BMC Public Health. 2009;9:249.

9. European Latin American Public Health Network. Lessons learned in a European-Latin American collaboration for developing postgraduate education in public health. Eur J Public Health. 2001;11(2):227-30

10. Kupfer L, Hofman K, Jarawan R, McDermott J, Bridbord K. Roundtable. Strategies to discourage brain drain. Bull World Health Organ. 2004;82(8):616-9; discussion 619-23.

11. Archer SL. The making of a physician-scientist--the process has a pattern: lessons from the lives of Nobel laureates in medicine and physiology. Eur Heart J. 2007;28(4):510-14.

12. Yewdell JW. How to succeed in science: a concise guide for young biomedical scientists. Part I: taking the plunge. Nat Rev Mol Cell Biol. 2008;9(5):413-16.

13. Coloma J, Harris E. From construction workers to architects: developing scientific research capacity in lowincome countries. PLoS Biol. 2009;7(7):e1000156.

14. Nchinda TC. Research capacity strengthening in the South. Soc Sci Med. 2002;54(11):1699-711.

15. Lansang MA, Dennis R. Building capacity in health research in the developing world. Bull World Health Organ. 2004;82(10):764-70.

16. Johnson MO, Subak LL, Brown JS, Lee KA, Feldman MD. An innovative program to train health sciences researchers to be effective clinical and translational research mentors. Acad Med. 2010;85(3):484-89.

17. Lansang MA, Olveda RO. Institutional linkages: strategic bridges for research capacity strengthening. Acta Trop. 1994;57(2-3):139-45.

18. Huamani C, Mayta-Tristán P. Producción científica peruana en medicina y redes de colaboración, análisis del Science Citation Index 2000-2009. Rev Peru Med Exp Salud Publica. 2010;27(3):315-25.

19. McCurdy L, Goode LD, Inui TS, Daugherty RM Jr, Wilson DE, Wallace AG, et al. Fulfilling the social contract between medical schools and the public. Acad Med. 1997;72(12):1063-70.

20. Norman JN, Brebner JA, Ruddick-Bracken H, Brebner EM, al-Ozairi SS. International collaboration in the development of postgraduate research training (692). Med Educ. 1998;32(1):82-84.

21. Kennedy TJ Jr. Graduate education in the biomedical sciences: critical observations on training for research careers. Acad Med. 1994;69(10):779-99.

22. Hotez PJ. Training the next generation of global health scientists: a school of appropriate technology for global health. PLoS Negl Trop Dis. 2008;2(8):e279.

23. Gray J, Armstrong P. Academic health leadership: looking to the future. Proceedings of a workshop held at the Canadian Institute of Academic Medicine meeting Québec, Que., Canada, Apr. 25 and 26, 2003. Clin Invest Med. 2003;26(6):315-26.

24. Costello LC. The effect of contemporary education and training of biomedical scientists on present and future medical research. Acad Med. 2009;84(4):459-63.

25. Ridde V, Mohindra KS, LaBossière F. Driving the global public health research agenda forward by promoting the 
participation of students and new researchers: perspectives from Quebec. Can J Public Health. 2008;99(6):460-65.

26. McPaul MJ. Issues in developing the medical scientist, part 2: fostering research among medical students (interview with Michael John McPhaul, MD). J Investig Med. 2004;52(5):292-5.

27. Kalfoglou AL, Sung NS. What inspires clinical research trainees and keeps them on the path? J Investig Med. 2002;50(6):408-11.

28. Villafuerte-Galvez J, Curioso WH, Miranda JJ. The role of medical students in the fight to control neglected tropical diseases: a view from Peru. PLoS Negl Trop Dis. 2008;2(9):e292.

29. Moreno-Borchart A. One problem at a time. Building research capacities in developing countries is necessary for economic success in the long term. But the numerous problems in doing so have to be solved for each country individually. EMBO Rep. 2004;5(2):127-30.

30. Omar M, Tarin E, Ashjaei K, Mirzoev T, Sheikh MR. Incountry capacity development of a training institute: an Iranian experience. J Health Organ Manag. 2007;21(6):51932.

31. Arriola-Quiroz I, Curioso WH, Cruz-Encarnación M, Gayoso O. Characteristics and publication patterns of theses from a Peruvian medical school. Health Info Libr J. 2010;27(2):148-54.

32. Guerra H. La beca de retorno de la Universidad Peruana Cayetano Heredia. Rev Peru Med Exp Salud Publica. 2010;27(3):326-36.

33. Marbán E, Braunwald E. Training the clinician investigator. Circ Res. 2008;103(8):771-72.

34. Ochola LI, Gitau E. Challenges in retaining research scientists beyond the doctoral level in Kenya. PLoS Negl Trop Dis. 2009;3(3):e345.

35. Garcia PJ, Curioso WH. Strategies for aspiring biomedical researchers in resource-limited environments. PLoS Negl Trop Dis. 2008;2(8):e274.

36. Fraser B. Hector Hugo García: promoting research on cysticercosis in Peru. Lancet. 2007;370(9599):1604.

37. Anand NP, Hofman KJ, Glass RI. The globalization of health research: harnessing the scientific diaspora. Acad Med. 2009;84(4):525-34.

38. Doumbo OK, Krogstad DJ. Doctoral training of African scientists. Am J Trop Med Hyg. 1998;58(2):127-32.

39. Reeder J. Health research and human development in Papua New Guinea. BMJ. 2000;321(7264):815-16
40. Sitthi-Amorn C, Somrongthong R. Strengthening health research capacity in developing countries: a critical element for achieving health equity. BMJ. 2000;321(7264):813-17.

41. Dovlo D. Managing the return and retention of national intellectual capacity. Bull World Health Organ. 2004;82(8):620-1.

42. Whelan A. Effectiveness of strategies for discouraging brain drain. Bull World Health Organ. 2004;82(8):619-20.

43. Gonzalez-Block MA. Health policy and systems research agendas in developing countries. Health Res Policy Syst. 2004;2(1):6

44. Walker SH, Ouellette V, Ridde V. How can PhD research contribute to the global health research agenda? Can J Public Health. 2006;97(2):145-48.

45. Kuruvilla S, Mays N, Pleasant A, Walt G. Describing the impact of health research: a Research Impact Framework. BMC Health Serv Res. 2006;6:134.

46. Chandiwana S, Ornbjerg N. Review of North-South and South-South cooperation and conditions necessary to sustain research capability in developing countries. J Health Popul Nutr. 2003;21(3):288-97.

47. Mayhew SH, Doherty J, Pitayarangsarit S. Developing health systems research capacities through north-south partnership: an evaluation of collaboration with South Africa and Thailand. Health Res Policy Syst. 2008;6:8.

48. Kitua AY, Corrah T, Herbst K, Nyirenda T, Agwale S, Makanga $\mathbf{M}$, et al. Strengthening capacity, collaboration and quality of clinical research in Africa: EDCTP Networks of Excellence. Tanzan J Health Res. 2009;11(1):51-54.

49. Arunachalam S. Does India perform medical research in areas where it is most needed? Natl Med $\mathrm{J}$ India. 1998;11(1):27-34.

50. Guerra H, Falconí E, Llanos-Cuentas A, Chang J. Investigación en medicina tropical y atención primaria a la salud en Perú. Salud Publica Mex. 1993;35(5):477-78.

51. Burstein Z. Homenaje al Dr. Ernst Georg Nauck. An Fac Med (Lima). 2003;64(4):274-78.

Correspondencia: Dr. Eduardo Gotuzzo

Dirección: Av Honorio Delgado 430, Urb. Ingeniería, Lima31, Perú.

Correo electrónico: eduardo.gotuzzo@upch.pe 